\title{
Limited capacity for memory tasks with multiple features within a single object
}

\author{
John Palmer ${ }^{1} \cdot$ Britt Boston $^{1} \cdot$ Cathleen M. Moore ${ }^{2}$
}

Published online: 6 May 2015

(C) The Psychonomic Society, Inc. 2015

\begin{abstract}
Memory for multiple features might be limited by the number of features, the number of objects, or both. To focus on the role of features, we tested memory for a variable number of features within a single object. Subjects studied a single ellipse that varied in four features: size, orientation, contrast, and position. We conducted two experiments that differed in how memory was tested. If performance is limited only by the number of objects to be remembered, there should be no effect of the number of relevant features within a single object. Instead, for both experiments, the proportion correct was lower when four features had to be remembered rather than one. The magnitude of these effects varied with the details of the two experiments. Although similar results have been reported for experiments using multiple objects, the present experiments are some of the first to have demonstrated such an effect for a single object. This result is inconsistent with theories in which visual memory has a discrete limit on the number of stored objects, and no limit on the stored features within an object. Instead, it seems likely that objects and features both play roles in limiting performance in memory tasks.
\end{abstract}

Keywords Visual working memory · Short-term memory · Attention

John Palmer

jpalmer@uw.edu

1 University of Washington, Seattle, WA, USA

2 University of Iowa, Iowa City, IA, USA
It has long been known that there are sharp limits on the amount of visual information that can be remembered for more than a fraction of a second (e.g., Phillips, 1974; Sperling, 1960). Luck and colleagues (Luck \& Vogel, 1997; Vogel, Woodman, \& Luck, 2001) described conditions in which this limit was determined by the number of visual objects (about four), with no apparent limit on the number of features within the objects. This result stands in contrast with other conditions that have revealed that the precision of feature judgments declines for even two objects, relative to one (Palmer, 1990; Zhang \& Luck, 2008).

In light of these observations, two hypotheses can be used to frame the debate about the nature of storage in visual memory, and by implication the source of memory limitations. According to the first hypothesis - the discrete-object limit one can store a fixed number of objects, regardless of their component features (Luck \& Vogel, 1997; Vogel et al., 2001; Zhang \& Luck, 2008). Memory is discrete, because an object is stored along with all its features, or it is not stored at all. According to the second hypothesis - the feature-precision limit - one can store a fixed amount of total information about the collection of features, regardless of their number and the objects to which they belong (Palmer, 1990; van den Berg, Shin, Chou, George, \& Ma, 2012; Wilken \& Ma, 2004). This hypothesis is related to the more general idea of a resource limit (Alvarez \& Cavanagh, 2004; Bays \& Husain, 2008). According to the feature-precision hypothesis, memory is not discrete, because the precision of information about features declines gradually with an increase in the number of remembered features. These two hypotheses are but a start. For example, one can have a discrete-slot model that represents features as well as objects, or one can have a continuous-precision model that is a function of object properties as well as feature properties. A variety of such hypotheses have been intensely debated (e.g., Anderson \& Awh, 2012; Fougnie, Asplund, \& Marois, 2010; Keshvari, van 
den Berg, \& Ma, 2013; Rouder et al., 2008; Zhang \& Luck, 2011; for general reviews, see Brady, Konkle, \& Alvarez, 2011, and van den Berg, Awh, \& Ma, 2014).

\section{Memory for a single object}

Here we focus on an unexplored, but potentially revealing, corner of this debate. Specifically, what is the effect of remembering multiple features for a single object? Is memory limited by the number of features that can be encoded, stored, and retrieved? Or is it unlimited? The case of a single object is special because no constraint is imposed by multiple objects. For example, it minimizes any concern that feature information is lost because it is not linked to the correct object (e.g., Wheeler \& Treisman, 2002). Testing a single object, therefore, allows for a more direct interpretation of any effect of the number of features.

According to the discrete-object hypothesis, performance should not be limited by the amount of to-be-remembered feature information beyond sensory limitations (e.g., acuity and crowding). In contrast, according to the featureprecision hypothesis, performance should decrease with an increase in the number of relevant features. Most previous studies have addressed this question only for multiple objects.

Only one study of which we are aware has examined perception and memory for one versus two features of a single object (Vogels, Eeckhout, \& Orban, 1988, Exp. 1). A briefly presented grating was varied in both orientation and spatial frequency. After an interval of $0.25 \mathrm{~s}$ a second grating appeared, and subjects judged "same" or "different" on the basis of an indicated relevant feature. The relevant feature was indicated either by a precue, presented $1.0 \mathrm{~s}$ before the first grating, or a postcue, presented $0.2 \mathrm{~s}$ after the second grating. Thus, with the precue, subjects only had to remember a single feature, whereas with the postcue, they had to remember both features. Performance was worse when they had to remember two features rather than one, a result consistent with a feature-precision limit.

It is natural to ask whether this result was due to a limit on perception or on memory. Much of the Vogels et al. (1988) study was based on conditions that minimized the role of memory. Indeed, Vogels et al. termed their effect one of "feature uncertainty." In contrast, we concentrate on their Experiment 1, which was an explicit memory task. Our focus is on performance in explicit memory tasks that are intended to reveal memory phenomena. The question of the relative contributions of perception and memory will be deferred to the General Discussion.

\section{Two models}

We addressed the question of memory for multiple features in the context of two contrasting models of how processing is limited: unlimited capacity and fixed capacity. These two models serve as landmark alternatives within which to ask the question.

The unlimited-capacity model needs little introduction; according to it, the number of relevant features should have no effect in paradigms that probe memory for a particular feature. In contrast, fixed-capacity models predict an effect of the number of relevant features (Palmer, 1990). Fixed-capacity processing for features means that the total amount of information that can be processed about the features within a given amount of time is limited. As applied to memory, this implies that one can remember a large amount of information about a single feature or can remember less information about multiple features. If, for example, processing is allocated equally across the features, then for $n$ features, one would remember $1 / n$ of the amount of information about each feature, relative to the case with only a single relevant feature. Of course, more complicated versions of fixed-capacity processing models are possible, in which processing is allocated to different features in a weighted manner. Our specific fixed-capacity predictions are based on Shaw's sample size model and assumptions of equal weighting and Gaussian distributions for the feature representations (Palmer, 1990; Shaw, 1980). Details of the model and its predictions are provided in the Appendix.

The concept of "capacity" is used in multiple ways. Here we are emphasizing processing capacity, which is rooted in early theories of attention (Broadbent, 1958). This is distinct from the concept of storage capacity that is at the heart of some memory models (Miller, 1956). A fixed-capacity storage model is limited by something like the number of "slots" available for storage. A fixed-capacity processing model, on the other hand, is limited by the encoding, consolidation, and/ or retrieval processes that act on the relevant information. Such a processing model can predict effects of increasing the number of features from one to two, whereas a typical storage model with a fixed number of slots does not predict limitations until storage capacity is reached.

\section{Experiment 1}

In this first experiment, we used a procedure similar to that of Vogels et al. (1988) and Palmer (1990). The stimuli were single objects that varied along four different features. A postcue indicated which feature of a previously presented object was to be reported, and the number of to-be-reported features - or memory set size-was either one or four. This was achieved by manipulating whether the postcue was constant within a given block of trials (memory set size 1) or varied across trials (memory set size 4 ). When the postcue was constant, observers could commit only the relevant feature to memory, whereas when the postcue was unknown, all four features had to be committed to memory until the postcue 
appeared. The discrete-object hypothesis predicts no effect of memory set size in this experiment, because there was always only a single object.

\section{Method}

Subjects The four subjects were 20- to 40-year-old adults with normal or corrected-to-normal vision. All of the subjects were volunteers, and some received a small payment in compensation for their time. Subject B.B. was an author. The others were naive with regard to the purpose of the study.

Apparatus Luminance-calibrated displays were presented on a video monitor with a $67-\mathrm{Hz}$ refresh rate and 640 -by-480 resolution. Viewed from a distance of $61 \mathrm{~cm}$, the center-tocenter spacing of pixels in the center of the screen was 2 arcmin. Stimuli were presented against a background that appeared "white" with a luminance of $200 \mathrm{~cd} / \mathrm{m}^{2}$.

Stimuli The stimuli were based on an ellipse with a 2:1 aspect ratio. Four features were varied: size, orientation, contrast, and horizontal position (relative to fixation). The standard stimulus had a size of 120 arcmin (major axis), an orientation of $45^{\circ}$, a contrast of $70 \%$, and a position at fixation. The variable stimulus varied in all four features across trials. For a given trial, each feature took on one of two possible values that either increased or decreased the value in the standard stimulus. This yielded 16 possible stimuli (see Fig. 1). The frame around each ellipse in the figure is used to illustrate the

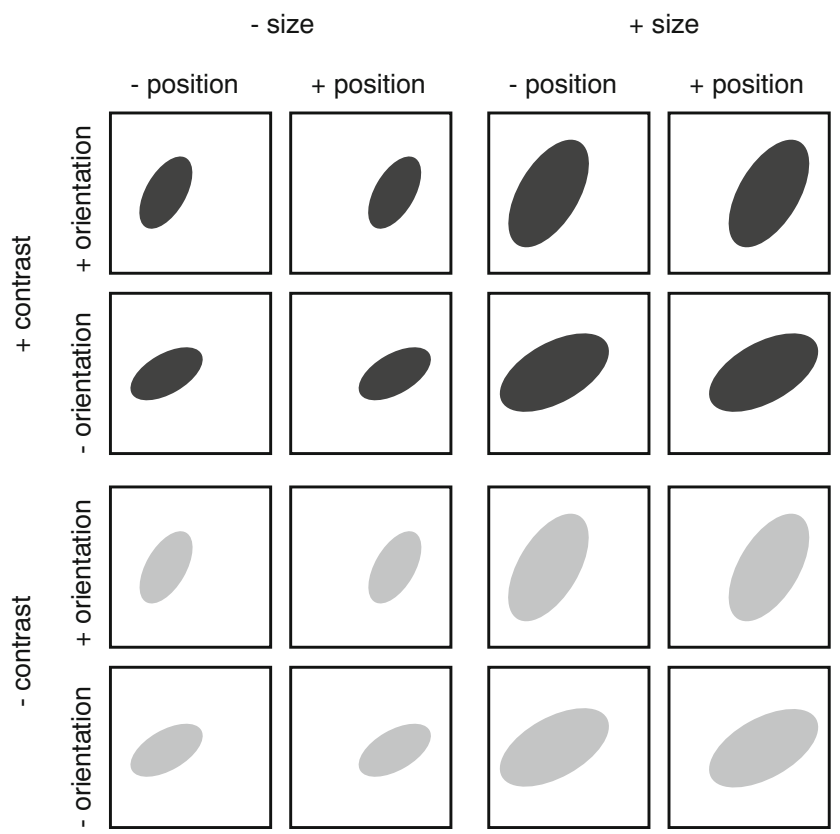

Fig. 1 Schematic illustration of the 16 possible stimuli in Experiment 1. The ellipses varied in size, orientation, contrast, and horizontal position. The frames are used here only to show the change in horizontal position and was not present in the experiment change in horizontal position and was not present in the experiment. The differences among the stimuli in this figure are exaggerated relative to the differences in the experiment. The particular values were adjusted for each subject on the basis of pilot data, so that the discrimination performance for each feature was in the range of $65 \%-85 \%$ correct. For example, Subject B.B. used size changes of \pm 2 arcmin, orientation changes of $\pm 1^{\circ}$, contrast changes of $\pm 5 \%$, and position changes of \pm 10 arcmin.

Procedure The procedure for a single trial is illustrated in Fig. 2. An initial fixation was followed by a first stimulus for $0.1 \mathrm{~s}$, a blank interval of $1 \mathrm{~s}$, a second stimulus for $0.1 \mathrm{~s}$, a blank interval of $1 \mathrm{~s}$, and a postcue until response. The postcue indicated the relevant feature through a label such as "size." For the cued feature, subjects used a keypress to indicate the relative magnitude of the second stimulus relative to the first. For example, was the second ellipse larger or smaller than the first? Subjects were instructed that for orientation, increasing "magnitude" corresponded to clockwise changes in orientation, and for location, increasing "magnitude" corresponded to leftward shifts. As is illustrated in Fig. 2, the two ellipses always varied in all four features. The second stimulus was always the standard. Despite the second stimulus

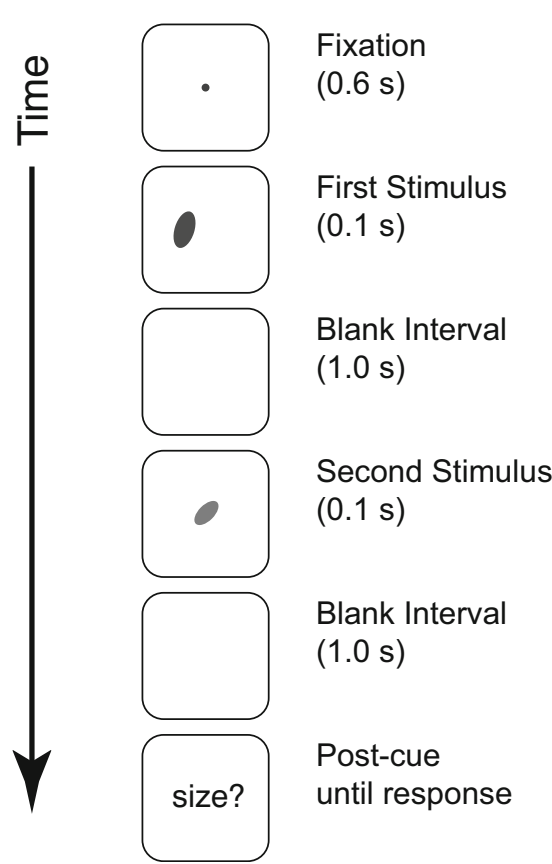

Fig. 2 Schematic illustration of the display sequence in Experiment 1. A first stimulus display was followed by a second stimulus display, and then a postcue indicating the relevant feature. For this illustration, the relevant feature is size, and the correct answer is that the second display is smaller than the first. For conditions with a single relevant feature (memory set size 1), only a single feature was tested for a block of trials. For conditions with four relevant features (memory set size 4), different features were tested within a block. Thus, one had to remember only one feature in memory set size 1 , and all four features in memory set size 4 
never changing, subjects often reported basing their decisions on a comparison of the two stimuli, because comparing the first to the second stimulus yielded better performance than did comparing the second stimulus to a long-term memory standard. For discussions of the pros and cons of this design, see Dyjas, Bausenhart, and Ulrich (2012) and Thiele, Pratte, and Rouder (2011).

Design The number of relevant features (memory set size) was either one or four. For memory set size 1, the to-be-reported feature was the same for a block of trials and was indicated by an instruction at the beginning of the block (e.g., "size" or "orientation"). For this condition, the subject knew to remember only that one feature. For memory set size 4, the to-be-reported feature varied from trial to trial. Thus, the subject had to remember all four features. The displays were drawn from identical sets of possible displays, regardless of the number of relevant features. After practice, eight sessions were run with 512 trials per session, resulting in 2,048 trials per set size per subject.

\section{Results and discussion}

The results are shown in Fig. 3, which plots the proportions correct against the numbers of relevant features (memory set size). Performance was worse when more features had to be perceived and remembered. The mean proportions correct were $.84 \pm .01$ with one feature and $.69 \pm .01$ with four features, for a reliable difference of $.14 \pm .01, t(3)=11.9$, $p<.001$. This difference was found for all features (size, $.15 \pm .02$; orientation, $.12 \pm .02$; contrast, $.17 \pm .02$; position, $.14 \pm .03)$.

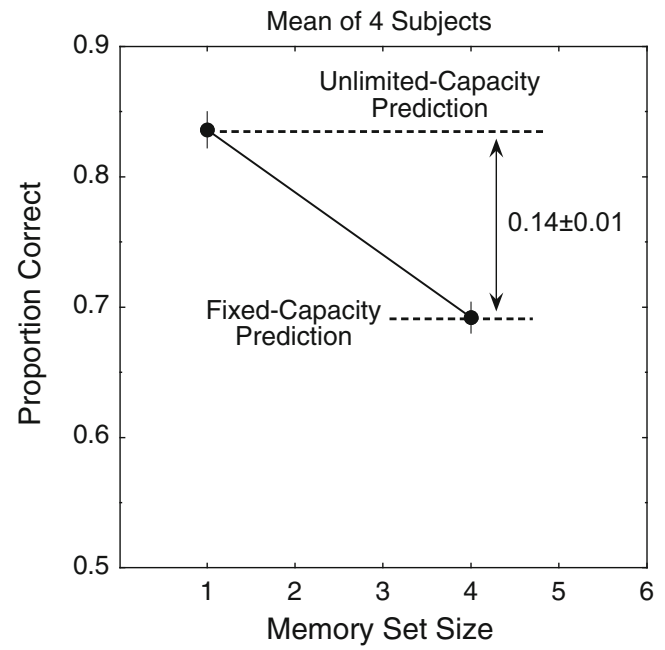

Fig. 3 Results of Experiment 1. Proportions correct are shown as a function of memory set size (the number of relevant features). Performance declined for the larger memory set size, and the effect matched that predicted by fixed-capacity models
Consider theories of visual working memory in which the discrete-object limit is the only limit on memory. When there is only one object, the processing of multiple features should be independent and thus should have unlimited capacity. As can be seen in the Fig. 3, the data from this experiment fall closer to what is predicted for fixed-capacity processing than for unlimited-capacity processing. Thus, this result is inconsistent with the discrete-object limit being the only limit on memory.

\section{Experiment 2}

In this second experiment, we pursued the same question with several changes in procedure to achieve three goals. First, in order to reduce the potential for condition-specific strategies, we mixed set sizes within blocks of trials, rather than having it blocked. Second, we made the experiment more similar to other visual memory experiments (e.g., Luck \& Vogel, 1997; Vogel et al., 2001) by increasing the duration of the study and the test display, and by presenting the postcue with the test display. Third, rather than a single memory standard throughout the experiment, we used multiple standards - often called a roving standard (e.g., Bull \& Cuddy, 1972; Harris, 1952). The purpose of the roving standard is to minimize the use of categorization strategies. For example, in Experiment 1 one might have remembered the single standard and judged the study display stimuli against that standard. In Experiment 2, subjects might still remember the center of the range and encode stimuli relevant to that point, but this strategy would be less effective. In addition, one can examine performance as a function of the standard to see whether stimuli at the ends of the range result in better performance than do stimuli in the center of the range. To foreshadow the result, we found no sign of such categorization effects.

\section{Method}

Subjects The six subjects were 20 - to 60 -year-old adults with normal or corrected-to-normal vision. All were volunteers, and some received a small payment in compensation for their time. Subject J.P. was an author. The others were naïve with regard to the purpose of the experiment.

Apparatus Luminance-calibrated displays were presented on a video monitor with a $75-\mathrm{Hz}$ refresh rate and 624-by-832 resolution. Stimuli were presented against a background that appeared "white" with a luminance of $108 \mathrm{~cd} / \mathrm{m}^{2}$.

Stimuli In Experiment 2 we used a roving standard. This change from Experiment 1 is illustrated in Fig. 4, which compares this aspect of the designs of Experiments 1 and 2 in panels A and B. In Experiment 1, each feature of the study 


\section{(a) Design of Experiment 1}

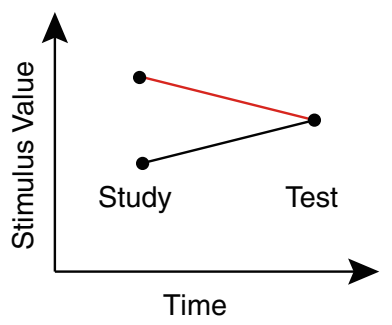

Test

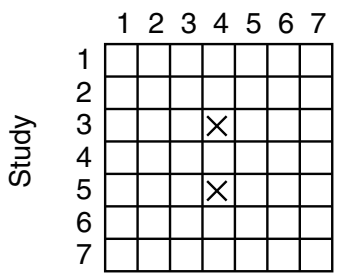

(b) Design of Experiment 2
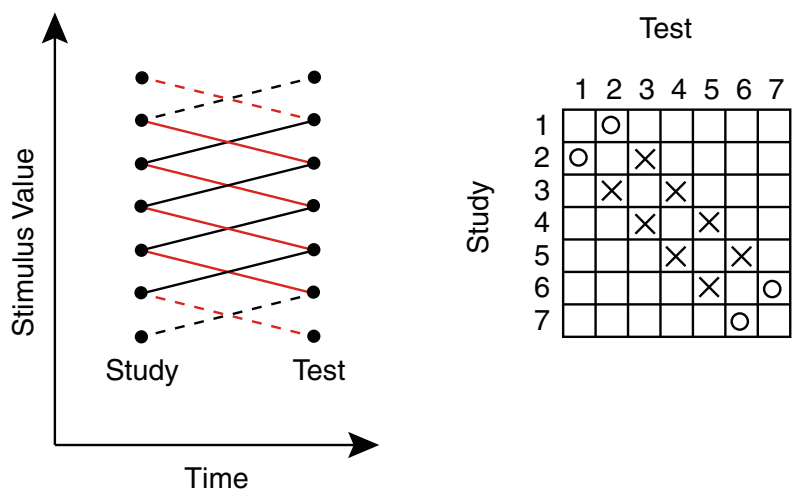

Fig. 4 The designs of Experiments 1 and 2 are shown in Panels A and B, respectively. On the left side is a graph of the possible stimulus values in the study display, linked to the possible values used in the following test display. On the right is the same information in a matrix format. In brief, in Experiment 1 we used a fixed standard for the test display, whereas in Experiment 2 we used a roving standard with many possible values

display could have two possible values, whereas the test display always had a single, standard value. This is illustrated on the left side of the figure by a graph that connects the possible values of a feature in the study and text displays: color lines for increasing values, and black lines for decreasing values. The possible stimulus values (e.g., size) are on the ordinate, and the different displays are distinguished on the abscissa by when they occur in time. An alternative representation is shown on the right, where the possible study and test value features are shown in an array: a single test value is paired with two possible study values.

Panel B shows the design of Experiment 2. Now there are seven possible values of both the study and test stimuli. The graph on the left shows how the feature value of the study is always followed by a test value that is one step "more" or "less" than the study value. Of course, the values at the ends of the range can be followed by only one possible test. These end-of-range cases are marked by dashed lines. The same design is shown in the array on the right. Now the end-ofrange cases are shown by circles, and the other cases are shown by " $\times$ " characters. This design has 12 possible stimulus pairs, which reduces to six possible stimulus pairs $(1,2),(2$, $3), \ldots,(6,7)$ if one ignores order. In the analysis of standards, these six pairs are referred to as Standards 1-6. These pairs were shown with equal probabilities.

As before, the stimuli were based on an ellipse with a 2:1 aspect ratio, and four features were varied: size, orientation, contrast, and horizontal position. To allow for the use of roving standards, the ellipses could have seven different values on the four features: The centers of these values were a size of $120 \mathrm{~min}$ of visual angle (major axis), an orientation of $45^{\circ}$, a contrast of $55 \%$, and a position at fixation. For three subjects, the steps between stimuli were $4 \operatorname{arcmin}$ for size, $3^{\circ}$ for orientation, $10 \%$ for contrast, and 10 arcmin for position. To roughly equate performance, the other three subjects had smaller steps.

Procedure Another change in this second experiment was that the number of relevant features was manipulated by using a precue that varied on every trial (see Fig. 5). The precue indicated the to-be-remembered feature(s) with a label. The label either indicated a single feature (e.g., "size") or indicated by "no cue" that all four features had to be remembered.

The procedure for a single trial is illustrated in Fig. 5. An initial fixation and cue display was presented for $1 \mathrm{~s}$. After an interval of $1 \mathrm{~s}$, the study stimulus was presented for $1 \mathrm{~s}$. This

$\begin{array}{cc}\text { Single } & \text { Four } \\ \text { Relevant } & \text { Relevant } \\ \text { Feature } & \text { Features }\end{array}$

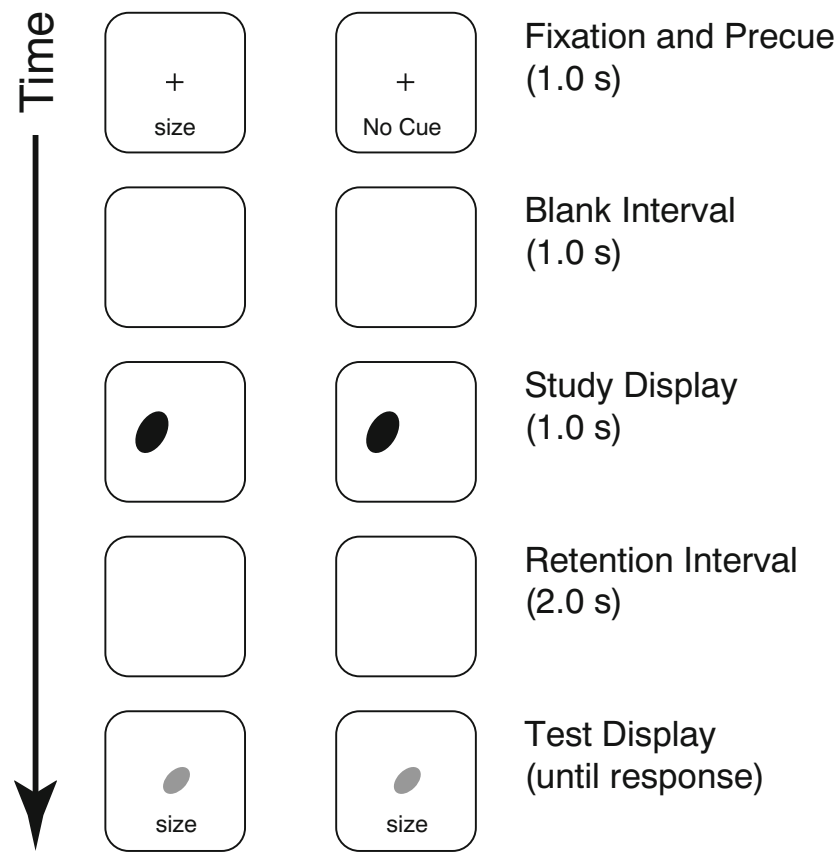

Fig. 5 Schematic illustration of the display sequence in Experiment 2. The two memory-set-size conditions are shown in the two columns. Trials began with a fixation and precue display. This was followed by a study and then a test display. The test display also contained a postcue and was present until response. For this illustration, the relevant feature is size, and the correct answer is that the second display is smaller than the first 
was followed by a retention interval of $2 \mathrm{~s}$ and by a test display that remained present until response. The test display contained both a comparison stimulus and a postcue indicating the relevant feature. Observers used a keypress to indicate the relative magnitude of the cued feature in the second display as compared to the first. For example, was the size of the second ellipse more or less than the first? As is illustrated in the figure, the two ellipses always varied in all four features. After practice, ten sessions were run with 192 trials per session, resulting in 960 trials per set size per subject.

The details of this procedure include three more differences between this experiment and Experiment 1. First, the study display was $1 \mathrm{~s}$ rather than $0.1 \mathrm{~s}$. Second, the test display was presented until response rather than for $0.1 \mathrm{~s}$. And third, the postcue indicating the to-be-reported feature was presented simultaneously with the test display, rather than following it by a second. All of these changes made this experiment more similar to other visual memory experiments rather than to a sequential-comparison experiment, on which the design of Experiment 1 was based. Specifically, in Experiment 2, the test stimulus was made more visible so that the roles of perception and memory for the study stimulus were emphasized. In contrast, the design of the first experiment had kept the roles of the two stimuli more symmetric.

\section{Results}

The results of Experiment 2 are shown in Fig. 6. It plots proportions correct as a function of the number of to-beremembered features (memory set size): Performance was worse when more features were relevant. The mean proportions correct were $.772 \pm .019$ with one relevant feature and $.750 \pm .014$ with four relevant features, for a reliable difference

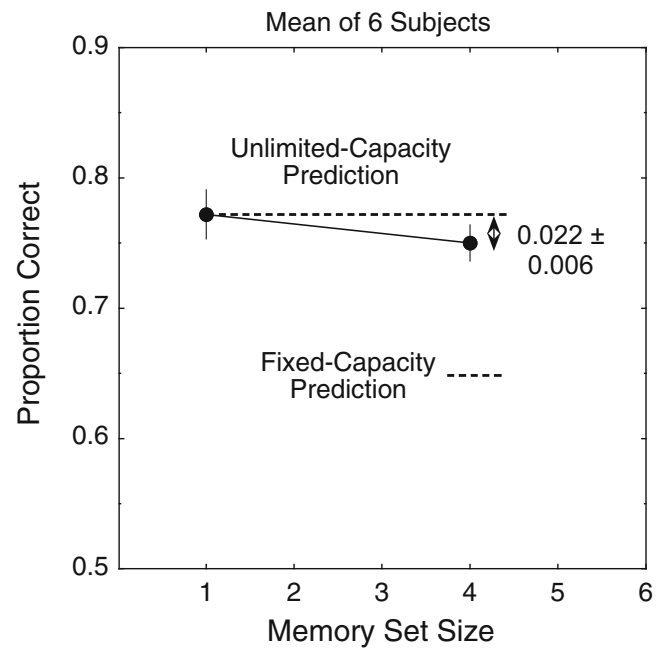

Fig. 6 Results of Experiment 2. Proportion correct is shown as a function of memory set size (the number of relevant features). Performance declined for the larger memory set size, and the difference was reliable of .022 $\pm .006, t(5)=3.6, p<.01$. Considering each feature separately, this difference was present but not reliable (size, $.01 \pm .01$; orientation, $.02 \pm .02$; contrast, $.03 \pm .03$; position, $.04 \pm .02)$.

To provide context, we show two predictions for the memory set size 4 condition, based on the performance observed in memory set size 1 . For unlimited capacity, performance with memory set size 4 should be identical to that in memory set size 1 . For fixed capacity, performance was predicted to be considerably worse for memory set size 4 . The observed difference, although reliable, was much less than that predicted by fixed capacity. Thus, unlike in Experiment 1, this result is inconsistent with a fixed-capacity model of processing multiple features.

In order to assess the possibility that observers adopted a categorical-judgment strategy, as we described in the introduction to this experiment, we looked at performance separately for each of the six standards (see Fig. 7). The data are shown for each of the four different feature types in separate panels, with a consistent order from "less" to "more." This ordering is clear for size and contrast. For orientation, the order is with respect to which is more clockwise, and for position, the value is with respect to which is more leftward. For example, size had standards of 108, 112, 116, 120, 124 , and 128 arcmin.
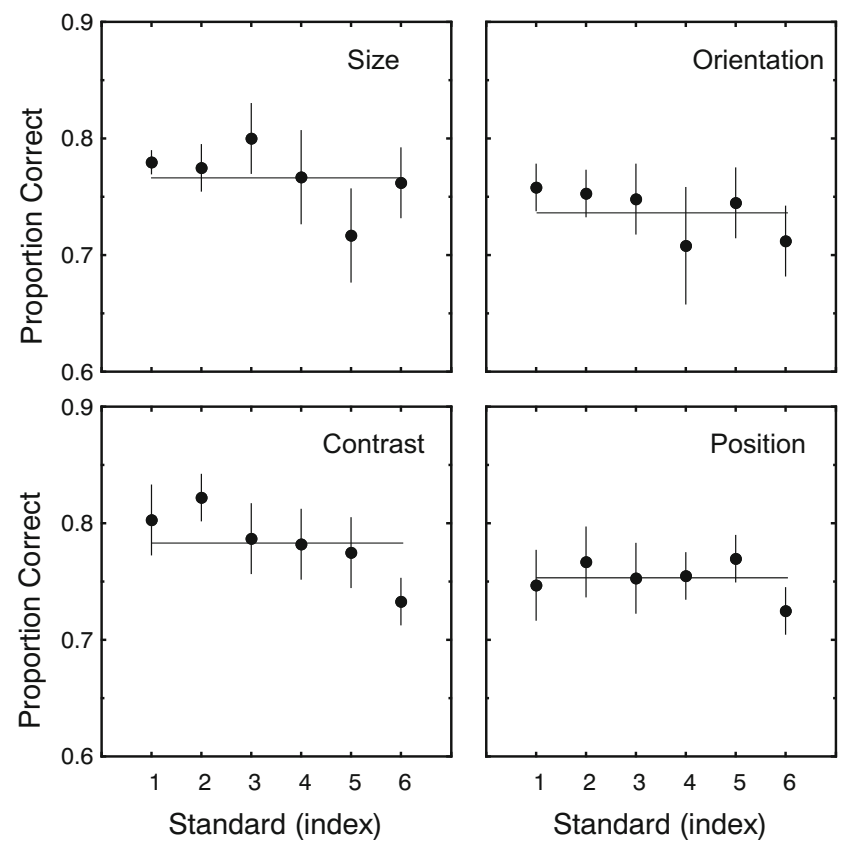

Fig. 7 The effect of the standard is illustrated for Experiment 2 with a separate panel for each feature. In each panel, the proportion correct is shown as a function of the standard's ordinal value. For comparison, a flat line is shown to represent no effect of the standard. In general there was no effect, although the figure hints at a decline for larger values of size and contrast. Of primary relevance here, there is no sign of improved performance for the standards at both ends of the range, as would be expected from a categorization strategy 
The figure shows no evidence of categorical judgments. There is no sign of a U-shaped function, such as would be expected from a categorical judgment. There is also no improvement for the standards at the end of the range. The hint of an improvement with size and contrast for larger standards is consistent with the commonly observed Weber's law, in which sensitivity declines for larger magnitudes.

\section{Discussion}

Up until now, we have focused on the issue of whether there is any effect of the number of features. In addition, it is desirable to measure the magnitude of this effect, which was much smaller in Experiment 2 than in Experiment 1. In the first experiment, the magnitude of the effect was close to the that predicted by fixed-capacity models, and in the second, it was a small fraction of that prediction. Thus, the second experiment allows one to reject the fixed-capacity model. Why the difference in Experiment 2?

Because several things were changed between these experiments, we do not know which was responsible. Perhaps the roving standard was important; perhaps the improved viewing conditions for the test was important. However, our primary concern is that the blocked design in Experiment 1 might have allowed for different strategies in the two set-size conditions. When only a single feature was relevant, observers might have judged that feature of a single object without waiting for the comparison object. In contrast, when multiple features were relevant, observers might have done as expected, and compared the postcued features present in the first and second objects. For Experiment 1, this strategy confounded the number of relevant objects (study vs. study and test) with the number of relevant features (1 vs. 4). This possibility undermines the feature interpretation of Experiment 1. In contrast, the mixed design combined with the roving standard made such confounding strategies impossible in Experiment 2, which is the stronger experiment for distinguishing the effects of features and objects.

In summary, Experiment 2 provides an improved test of the effect of the number of features, given a single object. Its design makes it unlikely that subjects used different strategies in the different conditions. This is our best evidence for an effect of the number of features.

\section{General discussion}

We found that varying the number of to-be-remembered features from a single object affected performance on a memory task. The key innovation of this study was that memory for multiple features was measured within a single object, rather than memory for features across multiple objects (see also Vogels et al., 1988). The special case of a single object is important, because it eliminates any possibility of the effects being mediated by the presence of multiple objects. For example, there was no opportunity for effects to arise from interference between multiple objects (e.g., McConnell \& Quinn, 2000) or from confusion regarding which features went with which objects (e.g., Wheeler \& Treisman, 2002). In general, using a single object is a way to study the effects of having to remember multiple features, with no possibility of confounding effects due to multiple objects. That the number of to-be-remembered features affected memory performance with a single object indicates that feature memory does not have unlimited capacity, and thus a discrete-object limit on visual memory cannot be the only limitation in visual memory. In addition, the relatively small effects found in Experiment 2 allow one to reject the fixed-capacity model, as well. The effect of the number of features falls between the two extreme models.

The following discussion has four parts. The first part relates our study of a single object to the larger literature on multiple objects. The next two parts address the theoretical interpretation of the effect of the number of relevant features, and the fourth part discusses the possible reasons for the difference in the magnitudes of the effects found in Experiments 1 and 2 .

\section{Effect of the number of features with multiple objects}

An influential study on visual working memory (Luck \& Vogel, 1997) came to a contrasting conclusion, that visual working memory is not limited by the number of to-beremembered features, but only by the number of objects in which those features are instantiated. In those experiments, subjects were presented with brief study displays of two to six colored lines, followed $0.9 \mathrm{~s}$ later by a test display that was either unchanged or different for one object. The subjects responded either "same" or "different" to the test display. In different blocks of trials, the subjects were instructed to detect changes in color, orientation, or both. Performance decreased as the number of objects increased, but little if any effect was apparent of the number of relevant features on performance. This result with multiple objects is in apparent conflict with the present study.

Later studies, however, have indicated that features do play a role (Cowan, Blume, \& Saults, 2013; Davis \& Holmes, 2005; Marshall \& Bays, 2013; Olson \& Jiang, 2002; Wheeler \& Treisman, 2002; Xu, 2002). A particularly simple demonstration of an effect of the number of relevant features was reported in Fougnie et al. (2010). They presented three stimuli that varied in color and orientation, and probed the memory of one feature in one object. Subjects had to report the relevant feature by using the adjustment method of Wilken and Ma (2004; see also Zhang \& Luck, 2008, 2011), which allows for estimates of both the precision of memories and the 
number of items remembered. In different blocks of trials, subjects had to remember the color, the orientation, or both. Increasing the number of relevant features resulted in a decrease in precision but no effect on the number of remembered objects. Fougnie and colleagues (Fougnie et al., 2010; see also Fougnie, Cormiea, \& Alvarez, 2013) suggested a hybrid model in which visual memory is limited both by the number of tobe-remembered objects and, separately, by the number of tobe-remembered features. The number of objects influences both storage capacity and precision, whereas the number of features influences only precision. The present results are also consistent with such a hybrid model, in that we found that processing was limited by the number of to-be-remembered features. Our single-object experiments do not speak to whether or not a further limit is imposed by the number of to-be-remembered objects.

\section{The nature of the capacity limits: Storage capacity versus processing capacity}

The capacity limitations observed in the present study might concern processing capacity, in the sense of the amount of information processed per display (Broadbent, 1958), rather than storage capacity, defined by the number of objects per display (Miller, 1956). Discussions regarding the capacity limitations of visual memory have often focused on limitations in the storage of visual information. This is perhaps a natural focus when thinking about memory. But, it is important to also consider possible processing limitations on the encoding, maintenance, and retrieval of visual information (see Cowan \& Morey, 2007; Fougnie \& Marois, 2009).

For an intuition regarding a precision limit due to limited processing capacity, consider Shaw's (1980) sample size model, which was the basis for our fixed-capacity predictions. According to this model, one can make $n$ samples of a stimulus representation per unit time. For a single relevant feature, these samples can all be on one feature. For four relevant features, the samples must be divided, which results in a less precise estimate for the individual features. For the case of evenly divided samples, the variance of the estimate is inversely related to the number of samples, and thus the standard deviation doubles when the memory set size increases by a factor of 4 . Thus, the capacity limits observed in this study could reflect a processing capacity limitation like that captured in this model, which would manifest as a limit on the precision of the representation of the visual information. This sampling intuition is a start, but it does not speak to what specific aspect of processing is limited: Is it encoding, maintenance, retrieval, or all of the above?

A specific processing-capacity hypothesis has been suggested for the effect of multiple features by Bays, Gorgoraptis, Wee, Marshall, and Husain (2011). They suggested a combination of a memory storage limit for objects and a memory encoding limit for the features within an object. To test this idea, they varied both stimulus duration and the numbers of features and objects, and found results consistent with this two-component hypothesis. Recently, this idea has been elaborated by Sewell, Lilburn, and Smith (2014).

A discrete-object limit can sometimes mimic a precision limit. Zhang and Luck (2008) suggested that the effects on precision that they inferred through a cue estimation procedure could be accounted for under a model that assumed a discrete-object limit on storage. Specifically, they proposed a model in which, when the memory set size is below the storage capacity limit, multiple copies of some objects can be stored, resulting in a measured improvement in precision. This solution does not work for the present study, because multiple copies of the (single) study object would improve the precision of all of the features of the object; there would be no advantage for specifying a single feature as relevant, rather than all four features.

The tendency to focus on storage limitations when considering memory might be a reason why the special case of a single object has been neglected. If one is focused only on possible limitations of memory storage, then it would be unlikely to consider a single-object case, because it would be unlikely to reveal the limits of the system.

\section{Memory or perception?}

The study-test paradigm used here was intended to measure visual memory, but might the observed effect of set size have been due to perception? This is a difficult question, and few studies have directly addressed it (e.g., Bays et al., 2011; Fougnie \& Marois, 2009; Mazyar, van den Berg, \& Ma, 2012; Sewell et al., 2014). Consider two opposing arguments. An argument for a perceptual account comes from the experiment of Mazyar et al. (2012), in which they found similar effects of the number of objects/features using matched search and memory paradigms (but see McLean, 1999). Mazyar and colleagues interpreted this result as being consistent with perceptual limits and no memory limits.

An argument against the perceptual account is that some experiments have shown no effect of the number of simultaneous objects/features using search tasks (e.g., Huang \& Pashler, 2005; Scharff, Palmer, \& Moore, 2011). The authors of these studies interpreted their results as showing no perceptual limits for these kinds of simple stimuli and tasks, and thus that memory must be the limit in study-test paradigms. Critically relevant to memory, this simultaneous-sequential procedure has been applied to the effect of multiple objects on memory performance by Sewell et al. (2014; see also Liu \& Becker, 2013; Mance, Becker, \& Liu, 2012). Sewell and colleagues found equal performance for simultaneous and sequential displays, consistent with no limit on perception, despite an effect of the total number of items to be remembered. 
The next step would be to use these methods to study the effect of the number of features with a single object. Sorting out this issue will be important, because one way to "save" a simple memory hypothesis is to attribute to perception any exception to the memory hypothesis.

\section{Experiment 1 compared to Experiment 2}

Experiments 1 and 2 yielded effects that differed markedly in magnitude. The effect in Experiment 1 was as large as that predicted by fixed-capacity models, whereas the effect in Experiment 2, though inconsistent with unlimited-capacity models, was much less than predicted by fixed-capacity models. Several procedural differences distinguished the two experiments, and therefore we cannot say with certainty what is the source (or are the sources) of the difference. We offered some speculations in the discussion of Experiment 2.

A broader view is that whatever the specific cause for the difference in effect sizes between the two experiments, it is an example of how the details of the procedure can change the effect of the number of relevant features. The task can change, for example, how the number of decisions varies with the number of features (see Busey \& Palmer, 2008, for an example of this in visual search). The task can simplify or complicate the retrieval process (see Anderson \& Bower, 1972, and Kintsch, 1970, for examples of this in verbal memory). The experiments reported here, for example, used a cued recognition task in which subjects had to judge a particular cued aspect of the study display. Specifically, one of four features was cued. In prior studies, an entire object (or set of objects) had been cued (e.g., Palmer, 1990). This cued recognition task was chosen because it minimizes the potential for contributions from decision and retrieval processes to the magnitude of the observed memory-set-size effects. The more common task of probe recognition, in which subjects judge whether a given stimulus was present anywhere in the memorized display (e.g., Mazyar et al., 2012) probably presents a more difficult retrieval and decision problem, because all of the elements of the study display are relevant to the response. But that task, in turn, presents a simpler decision and retrieval problem than does other change detection (e.g., Keshvari et al., 2013; Luck \& Vogel, 1997; Scott-Brown \& Orbach, 1998), which presents the subject with many decisions, since all of the elements of the study and test display are relevant. It remains to be worked out how the specific task influences the effect of the number of to-be-remembered features.

\section{Conclusions}

We measured performance in a memory task as a function of the number of relevant features in a single object. We found that increasing the number of relevant features decreased performance. This effect indicates that feature perception and memory do not have unlimited capacity, and therefore it rejects theories that assume only a discrete-object limit on performance. The present findings are consistent with hybrid models such as that offered by Fougnie et al. (2010) and Bays et al. (2011).

To address these questions further, we suggest that two special cases of visual working memory deserve particular study. The familiar case is: How many objects can one remember when the visual discriminations are relatively coarse? This case has been studied extensively. The other case is the one considered here: For a single object, how does the precision of feature memory depend on the number of relevant features? This case has received little study and has the potential to demonstrate limits on feature memory that have nothing to do with objects.

Author Note Experiment 1 was first presented in 1992 at the meeting of the Psychonomic Society in St. Louis, Missouri. In response to encouragement by our colleagues, it was renewed and presented in 2012 at a visual memory workshop in Portland, Oregon. We thank Anja Fiedler, Daryl Fougnie, Bjorn Hubert-Wallander, Geoff Loftus, Wei Ji Ma, Hongsup Shin, and anonymous reviewers for suggestions and criticism of an earlier version of this article.

\section{Appendix}

In this appendix, we describe in detail the unlimited- and fixed-capacity models that are used in this article. They are based on the sample size model (Shaw, 1980) and have been discussed in Palmer (1990), with the key derivation being given in the Perception Hypothesis section of the appendix of Palmer, Ames, and Lindsey (1993).

We use a notation similar to that in signal detection theory (Green \& Swets, 1966) and in related articles (e.g., Busey \& Palmer, 2008). In these theories, the judgment is based on a representation of a feature that corresponds to a single random variable, $U$. For the case of $n$ multiple features, there are corresponding random variables $U_{i}, i=1, \ldots, n$. We assume that these random variables are independent of one another and that they are identically distributed with a density function $f_{i}$ and a cumulative distribution function $F_{i}$. In addition, we assume that features that vary in their values on a single dimension correspond to the members of a shift family of the distribution function [i.e., $f(x-a)$, where $a$ is the shift in terms of the random variable]. For our experiment with a standard and comparison values of a given feature, we denote the distributions of the standard value as $f(x)$ and of the comparison value as $f(x-w \delta)$, where the amount of shift is given by $w \delta$, with $\delta$ being the physical description of the feature and $w$ the free parameter relating physical units to the signal/noise units of the representation. Finally, for numerical calculation of the 
specific predictions, we make the further assumption that the random variables are Gaussian.

Consider the case in which a single stimulus of $n$ features is presented, but only one of the features is relevant to the task (memory set size 1). We will focus on the design of Experiment 1 , in which each feature in the study display could take on either an increment $\delta_{+}$or a decrement $\delta_{-}$relative to the value in the study display. The subject's task was to indicate whether the relevant feature in the second display was larger or smaller than the one in the first display, which is a variation on a two-interval forced choice task. The first and second displays are indicated by $j=1,2$, and the $n$ features by $i=1, \ldots, n$, so that the random variables are $U_{i j}$. Given that the feature in the first display had a value of $\delta$ and that the standard value in the second display is denoted by 0 , the corresponding two random variables are $U_{i 1}(w \delta)$ and $U_{i 2}(0)$. According to this theory, the judgment is determined by which of these two random variables has the larger value on a given trial. The probability of judging the second display as being "larger" than the first is thus

$P($ "larger" $\mid \delta)=P\left[U_{i 2}(0)>U_{i 1}(w \delta)\right]$.

The probability correct for an increment is

$P($ correct $)=1-P\left(\right.$ "larger" $\left.\mid \delta_{+}\right)$.

Because the random variables are independent, one can solve this equation by taking the integral of the product of the density and the cumulative distribution of the two random variables

$P($ correct $)=1-\int_{-\infty}^{\infty} f(x) F(x-w \delta) d x$.

The probability correct for a decrement is

$P($ correct $)=P\left(\right.$ "larger" $\left.\mid \delta_{-}\right)$.

Given independence, this equation can be similarly solved as

$P($ correct $)=\int_{-\infty}^{\infty} f(x) F(x+w \delta) d x$.

Since these cases occur with equal probabilities, the combined probability correct is

$P($ correct $)=0.5\left[1-P\left(\right.\right.$ "larger" $\left.\left.\mid \delta_{+}\right)\right]+0.5\left[P\left(\right.\right.$ "larger" $\left.\left.\mid \delta_{-}\right)\right]$.
We can rewrite this equation using Eqs. 3 and 5:

$$
\begin{aligned}
P(\text { correct })= & 0.5\left[1-\int_{-\infty}^{\infty} f(x) F(x-w \delta) d x\right] \\
& +0.5\left[\int_{-\infty}^{\infty} f(x) F(x+w \delta) d x\right] .
\end{aligned}
$$

From this result, we can calculate performance for any given difference in features $\delta$.

Now consider the case in which one must perceive and remember multiple features. The key assumption of all postcue experiments is that the postcue allows one to retrieve just the relevant feature and to retrieve it independently of other features in memory. Thus, one can make a single decision based on the retrieved single value, rather than multiple decisions for multiple memories, as in probe recognition or change detection. Given unlimited capacity in both perception and memory, performance is the same for multiple features as for a single feature.

For the fixed-capacity model, we instead assume that a constant amount of information is remembered from the set of features (see Lindsay, Taylor, \& Forbes, 1968, for a discussion of information theory, capacity limits, and signal detection). The concept of fixed capacity is perhaps more easy to understand in the context of Shaw's (1980) sample size model. For this model, when only a single feature is relevant, all of the available samples can be directed toward that single feature. When multiple features are relevant, the samples are divided among the features. Given equal sampling of $n$ features, the variance of the random variable for a given feature is inversely proportional to the number of samples. Equivalently, the variance as a function of the number of relevant features $U(n)$ is proportional to the number of features:

$\operatorname{Var}[U(n)]=n \operatorname{Var}[U(1)]$

(see Palmer et al., 1993, for the derivation).

Given the use of standardized distributions such as $f(x)$, this change in the variance can be described as a scaling of the $x$ values by the standard deviation of the distribution. Specifically, let $f(x)$ describe the representation when all samples are directed at a single feature; when the samples are equally distributed over $n$ features, the representation becomes $f(x / \sqrt{n})$. Thus, the key prediction of Eq. 7 becomes the following for $n$ relevant features:

$$
\begin{aligned}
P(\text { correct })= & 0.5\left[1-\int_{-\infty}^{\infty} f\left(\frac{x}{\sqrt{n}}\right) F\left(\frac{x-w \delta}{\sqrt{n}}\right) d x\right] \\
& +0.5\left[\int_{-\infty}^{\infty} f\left(\frac{x}{\sqrt{n}}\right) F\left(\frac{x+w \delta}{\sqrt{n}}\right) d x\right] .
\end{aligned}
$$




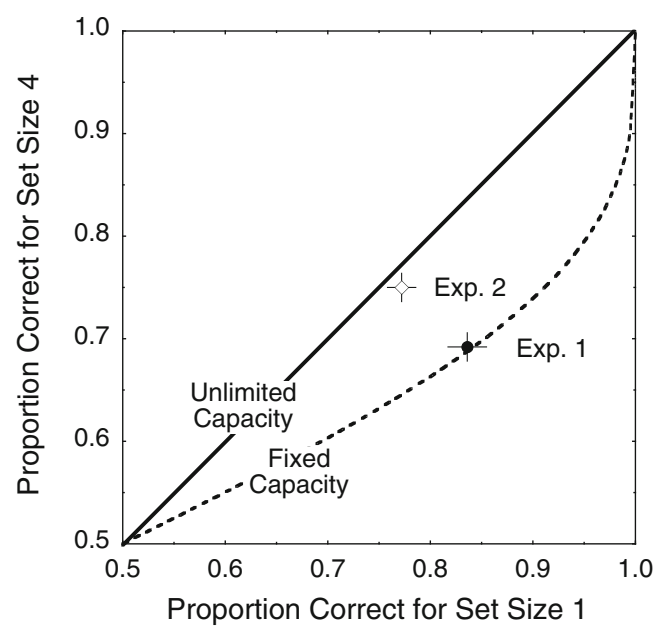

Fig. 8 This parametric plot shows the full range of predictions for the unlimited- and fixed-capacity models. It plots the proportion correct for set size 4 against the proportion correct for set size 1 . The positive diagonal shows equal performance as predicted by the unlimited-capacity model. The dashed curve shows the set-size effect predicted by the fixed-capacity model. Different points along the curve correspond to tasks of different difficulty. Also shown are the results of the two experiments

To provide a numerical example, assume Gaussian distributions and $w \delta=1$; then, the calculated probability correct for set size 1 is .760 , and for set size 4 it is .638. The complete set of predictions is shown in Fig. 8. It plots the proportions correct for set size 4 against the proportions correct for set size 1 . The positive diagonal denotes equal performance in the two conditions, as is predicted by an unlimited-capacity model. The dashed curve denotes the predictions for the fixedcapacity model. This curve is generated by varying $w \delta$ from 0 to 5 and calculating the probability correct from Eq. 9 for the two set sizes. Also shown are the results of the two experiments.

\section{References}

Alvarez, G. A., \& Cavanagh, P. (2004). The capacity of visual short-term memory is set both by visual information load and by number of objects. Psychological Science, 15, 106-111. doi:10.1111/j.09637214.2004.01502006.x

Anderson, D. E., \& Awh, E. (2012). The plateau in mnemonic resolution across large set sizes indicates discrete resource limits in visual working memory. Attention, Perception, \& Psychophysics, 74, 891-910. doi:10.3758/s13414-012-0292-1

Anderson, J. R., \& Bower, G. H. (1972). Recognition and retrieval processes in free recall. Psychological Review, 79, 97-123. doi: $10.1037 / \mathrm{h} 0033773$

Bays, P. M., \& Husain, M. (2008). Dynamic shifts of limited working memory resources in human vision. Science, 321, 851-854. doi:10.1126/science. 1158023

Bays, P. M., Gorgoraptis, N., Wee, N., Marshall, L., \& Husain, M. (2011). Temporal dynamics of encoding, storage, and reallocation of visual working memory. Journal of Vision, 11(10), 6. doi:10.1167/11.10.6

Brady, T. F., Konkle, T., \& Alvarez, G. A. (2011). A review of visual memory capacity: Beyond individual items and toward structured representations. Journal of Vision, 11(5), 4. doi:10. $1167 / 11.5 .4$

Broadbent, D. E. (1958). Perception and communication. New York, NY: Pergamon Press.

Bull, A. R., \& Cuddy, L. L. (1972). Recognition memory for pitch of fixed and roving stimulus tones. Perception \& Psychophysics, 11, $105-109$.

Busey, T., \& Palmer, J. (2008). Set-size effects for identification versus localization depend on the visual search task. Journal of Experimental Psychology: Human Perception and Performance, 34, 790-810. doi:10.1037/0096-1523.34.4.790

Cowan, N., \& Morey, C. C. (2007). How can dual-task working memory retention limits be investigated? Psychological Science, 18, 686688. doi:10.1111/j.1467-9280.2007.01960.x

Cowan, N., Blume, C. L., \& Saults, J. S. (2013). Attention to attributes and objects in working memory. Journal of Experimental Psychology: Learning, Memory, and Cognition, 39, 731-747.

Davis, G., \& Holmes, A. (2005). The capacity of visual shortterm memory is not a fixed number of objects. Memory \& Cognition, 33, 185-195.

Dyjas, O., Bausenhart, K. M., \& Ulrich, R. (2012). Trial-by-trial updating of an internal reference in discrimination tasks: Evidence from effects of stimulus order and trial sequence. Attention, Perception, \& Psychophysics, 74, 1819-1841.

Fougnie, D., \& Marois, R. (2009). Dual-task interference in visual working memory: A limitation in storage capacity but not in encoding or retrieval. Attention, Perception, \& Psychophysics, 71, 1831-1841. doi:10.3758/APP.71.8.1831

Fougnie, D., Asplund, C. L., \& Marois, R. (2010). What are the units of storage in visual working memory? Journal of Vision, 10(12), 27. doi:10.1167/10.12.27

Fougnie, D., Cormiea, S. M., \& Alvarez, G. A. (2013). Object-based benefits without object-based representations. Journal of Experimental Psychology: General, 142, 621-626.

Green, D. M., \& Swets, J. A. (1966). Signal detection theory and psychophysics. New York, NY: Krieger.

Harris, J. D. (1952). The decline of pitch discrimination with time. Journal of Experimental Psychology, 43, 96-99.

Huang, L., \& Pashler, H. (2005). Attention capacity and task difficulty in visual search. Cognition, 94, B101-B111. doi:10.1016/j.cognition. 2004.06.006

Keshvari, S., van den Berg, R., \& Ma, W. J. (2013). No evidence for an item limit in change detection. PLoS Computational Biology, 9(e1002927), 1-9. doi:10.1371/journal.pcbi.1002927

Kintsch, W. (1970). Models for free recall and recognition. In D. A. Norman \& H. A. Bernbach (Eds.), Models of human memory (pp. 331-373). New York, NY: Academic Press.

Lindsay, P. H., Taylor, M. M., \& Forbes, S. M. (1968). Attention and multidimensional discrimination. Perception \& Psychophysics, 4, $113-117$.

Liu, T., \& Becker, M. W. (2013). Serial consolidation of orientation information into visual short-term memory. Psychological Science, $24,1044-1050$

Luck, S. J., \& Vogel, E. K. (1997). The capacity of visual working memory for features and conjunctions. Nature, 390, 279281. doi: $10.1038 / 36846$

Mance, I., Becker, M. W., \& Liu, T. (2012). Parallel consolidation of simple features into visual short-term memory. Journal of Experimental Psychology: Human Perception and Performance, 38, 429-438. 
Marshall, L., \& Bays, P. M. (2013). Obligatory encoding of taskirrelevant features depletes working memory resources. Journal of Vision, 13(2), 1-13. doi:10.1167/13.2.21

Mazyar, H., van den Berg, R., \& Ma, W. J. (2012). Does precision decrease with set size? Journal of Vision, 12(6), 10. doi: $10.1167 / 12.6 .10$

McConnell, J., \& Quinn, J. G. (2000). Interference in visual working memory. Quarterly Journal of Experimental Psychology, 53A, 5367. doi:10.1080/027249800390664

McLean, J. E. (1999). Processing capacity of visual perception and memory encoding (Doctorial dissertation, University of Washington, Seattle). Dissertation Abstracts International, 60B, 3602.

Miller, G. A. (1956). The magical number seven, plus or minus two: Some limits on our capacity for processing information. Psychological Review, 63, 81-97. doi:10.1037/h0043158

Olson, I. R., \& Jiang, Y. (2002). Is visual short-term memory object based? Rejection of the "strong-object" hypothesis. Perception \& Psychophysics, 64, 1055-1067. doi:10.3758/BF03194756

Palmer, J. (1990). Attentional limits on the perception and memory of visual information. Journal of Experimental Psychology: Human Perception and Performance, 16, 332-350. doi:10.1037/00961523.16.2.332

Palmer, J., Ames, C. T., \& Lindsey, D. T. (1993). Measuring the effect of attention on simple visual search. Journal of Experimental Psychology: Human Perception and Performance, 19, 108-130. doi:10.1037/0096-1523.19.1.108

Phillips, W. A. (1974). On the distinction between sensory storage and short-term visual memory. Perception \& Psychophysics, 16, 283 290. doi:10.3758/BF03203943

Rouder, J. N., Morey, R. D., Cowan, N., Zwilling, C. E., Morey, C. C., \& Pratte, M. S. (2008). An assessment of fixed-capacity models of visual working memory. Proceedings of the National Academy of Sciences, 105, 5975-5979. doi:10.1073/pnas.0711295105

Scharff, A., Palmer, J., \& Moore, C. M. (2011). Extending the simultaneous-sequential paradigm to measure perceptual capacity for features and words. Journal of Experimental Psychology: Human Perception and Performance, 37, 813-833. doi:10.1037/ a0021440

Scott-Brown, K. C., \& Orbach, H. S. (1998). Contrast discrimination, non-uniform patterns and change blindness. Proceedings of the Royal Society B, 265, 2159-2166.
Sewell, D. K., Lilburn, D., \& Smith, P. L. (2014). An information capacity limitation of visual short-term memory. Journal of Experimental Psychology: Human Perception and Performance, 40, 2214-2242.

Shaw, M. L. (1980). Identifying attentional and decision-making components in information processing. In R. S. Nickerson (Ed.), Attention and performance VIII (pp. 106-121). Hillsdale, NJ: Erlbaum.

Sperling, G. (1960). The information available in brief visual presentation. Psychological Monographs: General and Applied, 74(11, Whole No. 498), 1-29.

Thiele, J. E., Pratte, M. S., \& Rouder, J. N. (2011). On perfect workingmemory performance with large numbers of items. Psychonomic Bulletin \& Review, 18, 958-963.

van den Berg, R., Shin, H., Chou, W. C., George, R., \& Ma, W. J. (2012). Variability in encoding precision accounts for visual short-term memory limitations. Proceedings of the National Academy of Sciences, 109, 8780-8785. doi:10.1073/pnas.1117465109

van den Berg, R., Awh, E., \& Ma, W. J. (2014). Factorial comparison of working memory models. Psychological Review, 121, 124-149. doi: 10.1037/a0035234

Vogel, E. K., Woodman, G. F., \& Luck, S. J. (2001). Storage of features, conjunctions, and objects in visual working memory. Journal of Experimental Psychology: Human Perception and Performance, 27, 92-114. doi:10.1037/0096-1523.27.1.92

Vogels, R., Eeckhout, H., \& Orban, G. A. (1988). The effect of feature uncertainty on spatial discriminations. Perception, 17, 565-577.

Wheeler, M. E., \& Treisman, A. M. (2002). Binding in short-term visual memory. Journal of Experimental Psychology: General, 131, 4864. doi:10.1037/0096-3445.131.1.48

Wilken, P., \& Ma, W. J. (2004). A detection theory account of change detection. Journal of Vision, 4(12), 11. doi:10.1167/4.12.11

$\mathrm{Xu}, \mathrm{Y}$. (2002). Limitations of object-based feature encoding in visual short-term memory. Journal of Experimental Psychology: Human Perception and Performance, 28, 458-468. doi:10.1037/00961523.28.2.458

Zhang, W., \& Luck, S. J. (2008). Discrete fixed-resolution representations in visual working memory. Nature, 453, 233-235. doi:10.1038/ nature 06860

Zhang, W., \& Luck, S. J. (2011). The number and quality of representations in working memory. Psychological Science, 22, 1434-1441. doi:10.1177/0956797611417006 